\title{
The Symmetry-Conforming Theory of Martensite Aging
}

\author{
Victor A. L'vov',a, Anna Kosogor ${ }^{1, a}$, Outi Söderberg ${ }^{2, b}$ \\ and Simo-Pekka Hannula ${ }^{2, c}$ \\ ${ }^{1}$ Department of Radiophysics, Taras Shevchenko University, 01601, Kyiv, Ukraine \\ ${ }^{2}$ Department of Materials Science and Engineering, Helsinki University of Technology, \\ P.O. Box 6200, Espoo FI-02015 TKK, Finland \\ avictorlvov@univ.kiev.ua, ${ }^{b}$ osoderbe@cc.hut.fi, 'simo-pekka.hannula@tkk.fi
}

\begin{abstract}
Keywords: Martensite aging. Martensite stabilization. Symmetry. Landau theory.
\end{abstract}
\begin{abstract}
The Landau theory has been developed for the description of martensite aging. The characteristic features of the theory are: i) the multicomponent non-scalar character of the order parameter describing the slow reconfiguration of lattice defects after martensitic transformation (MT); ii) the complete agreement with Symmetry-Conforming Short-Range-Order principle formulated by X. Ren and K. Otsuka; iii) the applicability to the different MT-s and various defects related to aging phenomena. The physical values interpreted as the components of internal stress, which stabilizes certain variant of martensitic phase, have been composed of the components of slow non-scalar order parameter. An applicability of the developed theory to the description of influence of aging on the MT temperature and yield stress was demonstrated.
\end{abstract}

\section{Introduction}

The aging of thermoelastic martensites is known as an important phenomenon resulting in the pronounced physical effects. Among these effects the stabilization of martensite is the most widely studied. The stabilization exhibits itself as the widening of the temperature range of martensitic phase and as the elevation of the yield stress and plateau-like segment at the stress-strain dependencies taken in this phase (see [1,2] and references therein). The martensite aging is often related to the reconfiguration of point defects, which is governed by the Symmetry-Conforming Short-Range-Order (SC-SRO) principle [1,2]. According to this principle the probabilities of finding of point defects in certain crystallographic positions possesses the same symmetry as the crystal symmetry. However, not only point defects but the defects of other types also contribute to the aging process.

An attempt of general theoretical description of aging effect in different shape memory alloys was undertaken in [3]. The special version of Landau theory was developed to derive the macroscopic equations for aging phenomena in two-dimensional crystal lattice. To this end the existence of one-component order parameter describing the slow aging processes was postulated. In spite of the positive ideas formulated in [3], the consideration of two-dimensional lattice makes its formal basis inadequate to SC-SRO principle, because the spontaneous deformation during MT occurs in three dimensions. Moreover, the martensitic phase includes differently oriented unit cells of crystal lattice. According to SC-SRO principle the equilibrium spatial distributions of point defects must be "fitted" to the lattice of different martensite variants. As so, the MT must be accompanied by redistribution of defects in three dimensions.

In the present article the Landau theory with multicomponent slowly varying (slow) order parameter is proposed for the description of martensite aging. The conformity with SC-SRO principle is considered the governing condition for the choice of slow order parameter. The suitability of proposed theory for the description of experimentally studied physical effects of martensite aging is demonstrated. In contrast to the model developed in [3] the present theory does 
not deny the possibility of stabilization of single-variant martensite and allows the stabilization of both multivariant and single-variant states.

\section{Results}

Landau Theory Describing the Martensite Stabilization. The Landau theory is successfully applied to thermoelastic martensitic transformations of cubic-tetragonal and cubic-rhombohedral types, which are described by the diagonal and non-diagonal strain tensor components $\varepsilon_{i k}$ $(i, k=x, y, z)$, respectively (see e.g. [4-7]). For the sake of certainty the Landau theory will be applied below to the description of aging of martensite formed as the result of cubic-tetragonal MT.

If the SC-SRO principle is valid, the slow process of reconfiguration of defects must follow the deformation of crystal lattice in the course of every martensitic transformation or martensite variants rearrangement, and vice versa, the every reconfiguration of defects must be accompanied by the local straining/stressing of the crystal lattice. This point can be introduced in the framework of Landau theory as the Principal Requirement of transformational equivalence of the order parameters describing deformation of crystal lattice and reconfiguration of crystal defects.

The Gibbs potential of the crystal with defects can be presented in the form

$$
G=F_{e}\left(u_{\alpha}\right)+F_{r}\left(\eta_{\alpha}\right)+F_{e r}\left(u_{\alpha}, \eta_{\alpha}\right)-3 \sigma_{1} u_{1}-\frac{1}{6}\left(\sigma_{2} u_{2}+\sigma_{3} u_{3}\right)
$$

where

$$
F_{e}=\frac{1}{2} c_{1} u_{1}^{2}+\frac{1}{2} c_{2}(T)\left(u_{2}^{2}+u_{3}^{2}\right)+\frac{1}{2} a_{2} u_{1}\left(u_{2}^{2}+u_{3}^{2}\right)+\frac{1}{3} a_{4} u_{3}\left(u_{3}^{2}-3 u_{2}^{2}\right)+\frac{1}{4} b_{4}\left(u_{2}^{2}+u_{3}^{2}\right)^{2}
$$

is the elastic energy $\left(c_{1}, c_{2}, a_{2}, a_{4}\right.$ and $b_{4}$ are the phenomenological parameters introduced in [6]), $F_{r}$ is the energy of defects reconfiguration in the course of MT, the term $F_{e r}$ describes the interrelation between the defects configuration and strains, The scalar value $u_{1}=\left(\varepsilon_{x x}+\varepsilon_{y y}+\varepsilon_{z z}\right) / 3$ and twocomponent value $u_{2}=\sqrt{3}\left(\varepsilon_{x x}-\varepsilon_{y y}\right), u_{3}=2 \varepsilon_{z z}-\varepsilon_{y y}-\varepsilon_{x x}$ are the basic functions of one-dimensional and two-dimensional irreducible representations of cubic group, respectively, the values $\sigma_{1}=\left(\sigma_{x x}+\sigma_{y y}+\sigma_{z z}\right) / 3, \sigma_{2}=\sqrt{3}\left(\sigma_{x x}-\sigma_{y y}\right)$ and $\sigma_{3}=2 \sigma_{z z}-\sigma_{y y}-\sigma_{x x}$ are the basic functions composed of stress tensor components $\sigma_{i k}$.

According to Principal Requirement the reconfiguration of crystal defects must be described by the one-component and two-component slowly variable basic functions of irreducible representations of cubic group $\eta_{1}(t)$ and $\eta_{2,3}(t)$; the reconfiguration energy polynomial can be obtained from Eq. 2 by substitution $u_{\alpha} \rightarrow \eta_{\alpha} \quad(\alpha=1,2,3)$ and appropriate denomination of polynomial coefficients. We intend to consider only those changes in the configuration of defects, which accompany the changes in martensitic structure, i.e. the spontaneous reconfiguration is not considered. Therefore, the third- and forth- order polynomial terms can be disregarded and simplified expression

$$
F_{r}=\frac{1}{2} \gamma_{1} \eta_{1}^{2}+\frac{1}{2} \gamma_{2}\left(\eta_{2}^{2}+\eta_{3}^{2}\right)
$$

can be used for reconfiguration energy. (The values $\gamma_{1,2}$ are phenomenological parameters).

In view of the Principal Requirement the bilinear combinations $\eta_{1} u_{1}, \eta_{2} u_{2}+\eta_{3} u_{3}$ of the order parameters $u_{\alpha}$ and $\eta_{\alpha}$ are invariant with respect to the cubic group so the energy $F_{e r}$ has a form 


$$
F_{e r}=-d_{1} \eta_{1} u_{1}-d_{2}\left(\eta_{2} u_{2}+\eta_{3} u_{3}\right)
$$

where $d_{1,2}$ are phenomenological parameters.

The single crystal whose Gibbs potential has the form Eq. 1 may be treated as two subsystems interrelated with each other, namely i) the atoms situated in the regular (proper) crystallographic positions; ii) the defects. As it is known, the subsystem i) quickly relaxes to its equilibrium state while subsystem ii) relaxes slowly, when its time evolution involves the diffusion of point defects and dislocation creep. The extremum conditions $\partial G / \partial \eta_{\alpha}=0$ result in the linear interrelation between the equilibrium values of quick and slow order parameters $\eta_{\alpha}^{(e q)}$ and $u_{\alpha}^{(e q)}$, respectively

$$
\eta_{1}^{(e q)}=d_{1} u_{1}^{(e q)} / \gamma_{1}, \quad \eta_{2,3}^{(e q)}=d_{2} u_{2,3}^{(e q)} / \gamma_{2}
$$

It can be concluded now that the fulfillment of Principal Requirement results in the linear interrelation between the strain tensor components characterizing a stationary deformation of crystal lattice and the equilibrium values of slow order parameter. This interrelation provides for the maintenance of SC-SRO principle: according to Eq. 5 the appearance of stationary strains in the crystal is mandatory followed by the slow reconfiguration of defects and vice versa, the reconfiguration of defects results in the straining of crystal lattice.

Conception of Stabilizing Internal Stress. The equation $\partial G / \partial u_{1}=0$ allows to express the $u_{1}$ variable through $u_{2,3}, \sigma_{1}$ and $\eta_{1}$. The substitution of expressed variable into Eq. 1 results only in the following renormalization of phenomenological parameters and stresses:

$$
c_{2}(T) \rightarrow c_{2}^{*}(T, t)=c_{2}(T)+3 a_{2} \sigma_{1}^{(e f f)}(t) / c_{1}, \quad b_{4} \rightarrow b_{4}^{*}=b_{4}-a_{2} / 2 c_{1}, \quad \sigma_{2,3} \rightarrow \sigma_{2,3}^{(e f f)}(t) .
$$

The effective stresses $\sigma_{\alpha}^{(e f f)}(t)$ are defined here as $\sigma_{\alpha}^{(e f f)}(t)=\sigma_{\alpha}+\sigma_{\alpha}^{(s)}(t)$, where the values

$$
\sigma_{1}^{(s)}(t)=d_{1} \eta_{1}(t) / 3, \quad \sigma_{2,3}^{(s)}(t)=6 d_{2} \eta_{2,3}(t)
$$

can be interpreted as the stresses induced in the course of adjustment of defects configuration to the symmetry of crystal lattice in the low temperature phase. It follows from Eqs. 5, 7 that the values $\sigma_{\alpha}^{(s)}(t)$ and $u_{\alpha}$ are of the same sign independently on the $d_{1,2}$ signs. The stress $\sigma_{\alpha}^{(s)}(t)$ stabilizes the low temperature phase hence. This Stabilizing Internal Stress (SIS) consists of the Stabilizing Pressure (SP) $\left(P^{(s)}(t)=-\sigma_{1}^{(s)}(t)\right)$ and Stabilizing Axial Stress (SAS) components $\left(\sigma_{2}^{(s)}\right.$ and $\left.\sigma_{3}^{(s)}\right)$.

The isotropic and axial internal stressing of martensite during the aging are two different factors, which contribute to martensite stabilization.

The idea of SIS opens a way of generalization of theoretical description of the aging phenomenon and related physical effects. First, SIS can be induced by the every slowly evolving defects and alloy structure elements, which induce the elastic straining of the crystal lattice (it should be remembered that the elastic strain is necessarily related to stress by Hook's law). The SIS may arise not only in the course of MT, but also during the other processes, such as generation of sessile dislocations during the deformation cycles, rearrangement of twinning dislocations or martensite variants, formation of self-accommodating groups, etc.

The Influence of Martensite Aging on Yield Stress. To describe the effect of aging on the pseudoelastic behavior of martensite one should determine the time dependence of slow order parameter components. In the simplest approach the generalized forces, which cause the time variation of slow order parameter components, may be considered linearly related to the derivatives of reconfiguration energy, i. e. $\dot{\eta}_{\alpha}=-\kappa_{\alpha} \partial F_{r} / \partial \eta_{\alpha}$. This equation must be resolved with the 
boundary conditions $\eta_{\alpha}(0)=0$ and $\eta_{\alpha}(\infty)=\eta_{\alpha}^{(e q)}$. As a result, the time dependencies of slow order parameter components and stabilizing stresses Eq. 7 are expressed as

$$
\eta_{\alpha}=\eta_{\alpha}^{(e q)}\left[1-\exp \left(-t / \tau_{\alpha}\right)\right], \quad \sigma_{\alpha}^{(s)}(t)=\sigma_{\alpha}^{(s)}(0)\left[1-\exp \left(-t / \tau_{\alpha}\right)\right],
$$

where $\tau_{1}=1 / \kappa_{1} \gamma_{1}$ and $\tau_{2,3}=1 / \kappa_{2,3} \gamma_{2}$ are the relaxation times of these components, $\sigma_{1}^{(s)}(0)=d_{1}^{2} u_{1}^{(e q)} / 3 \gamma_{1}, \sigma_{2,3}^{(s)}(0)=6 d_{2}^{2} u_{2,3}^{(e q)} / \gamma_{2}$.

External axial stressing of $z$-variant of martensite with $c / a<1$ in $y$-direction results in the transformation of this variant into $y$-variant. In this case $\sigma_{3}=-\sigma_{y y}>0$ and the dependence of yield stress on the aging time $t=t_{0}=$ const can be found from the equation

$$
\left|\sigma_{y y}\right| \equiv \sigma_{c}\left(t_{0}\right)=\sigma_{c}(0)-\sigma_{3}^{(s)}\left(t_{0}\right)
$$

When $c / a<1$ the SIS is negative, and so, the aging elevates the absolute value of yield stress.

The Influence of Martensite Aging on MT Temperature. Let $T_{1,2}$ are the temperature values found from the equations $c_{2}^{*}\left(T_{1}, 0\right)=c_{t}^{*}(0)$ and $c_{2}^{*}\left(T_{2}, 0\right)=0$, where $c_{t}^{*}(t) \equiv\left[a_{4}(t)\right]^{2} / 4 b_{4}^{*}$. For the aged alloy the temperature-dependent coefficient $c_{2}^{*}\left(T, t_{0}\right)$ differs from its initial value $c_{2}^{*}(T, 0)$ because of the presence of SP (see Eq. 6 and remeber that $\sigma_{1}^{(s)}(t)=-P^{(s)}(t)$ ). This coefficient can be approximated by the formula $c_{2}^{*}\left(T, t_{0}\right)=c_{t}^{*}\left(t_{0}\right)\left[T-T_{2}^{*}\left(t_{0}\right)\right] /\left[T_{1}^{*}\left(t_{0}\right)-T_{2}^{*}\left(t_{0}\right)\right]$, where the values $T_{1}^{*}\left(t_{0}\right), T_{2}^{*}\left(t_{0}\right)$ are the lability temperatures of the aged tetragonal and cubic phases, respectively. These temperatures can be found from the equations $c_{2}^{*}\left(T_{1}^{*}, t_{0}\right)=c_{t}^{*}\left(t_{0}\right)$ and $c_{2}^{*}\left(T_{2}^{*}, t_{0}\right)=0$ using the Eq. 6. The time-dependent shifts of lability temperatures caused by SP proved to be expressed by the formula

$$
\Delta T_{M}^{i s o t r}\left(t_{0}\right)=c_{t}(0)^{-1}\left[T_{2} c_{2}^{*}\left(T_{1}, t_{0}\right)-T_{1} c_{2}^{*}\left(T_{2}, t_{0}\right)\right]-T_{2}
$$

and equal to each other.

For the determination of the axial stress contribution to the shift of characteristic MT temperature the theoretical stress-strain diagram should be considered. However, this value can be estimated in a simplified manner from the obvious relationship

$$
\Delta T_{M}^{\text {anis }}\left(t_{0}\right)=\sigma_{z z}^{(s)}\left(t_{0}\right)\left(d T_{M}^{\text {anis }} / d \sigma_{z z}\right),
$$

where $\sigma_{z z}^{(s)}<0$. The value of derivative $\left(d T_{M}^{\text {anis }} / d \sigma_{z z}\right)$ can be taken from the experiments on stressinduced MT-s performed in compressive mode.

An example of slow order parameter. In the particular case of point defects the functions $\eta_{\alpha}(t)$ can be composed of the probabilities of finding of the defect in the certain crystal cites. As an example let us consider the cubic lattice with the center of inversion (see Fig. 1). Let $P_{1}, P_{2}$, and $P_{3}$ be the nonequilibrium probabilities of occupation of the certain regular sites by the point defect. Suppose that the aging effect be related mainly to the diffusion of point defects between these sites.

The symmetry operations of cubic group interchange the atomic sites, and hence, permute the subscripts, which enumerate probabilities. This permutation results in the transformation rules for probabilities. The Table 1 shows the transformation of probabilities and diagonal stress tensor components under the action of generating elements of cubic group (Î symbolizes the inversion operation, $4_{y}$ and $4_{z}$ denote the operations of $90^{\circ}$-turns around $y$-and $x$-axes respectively). 


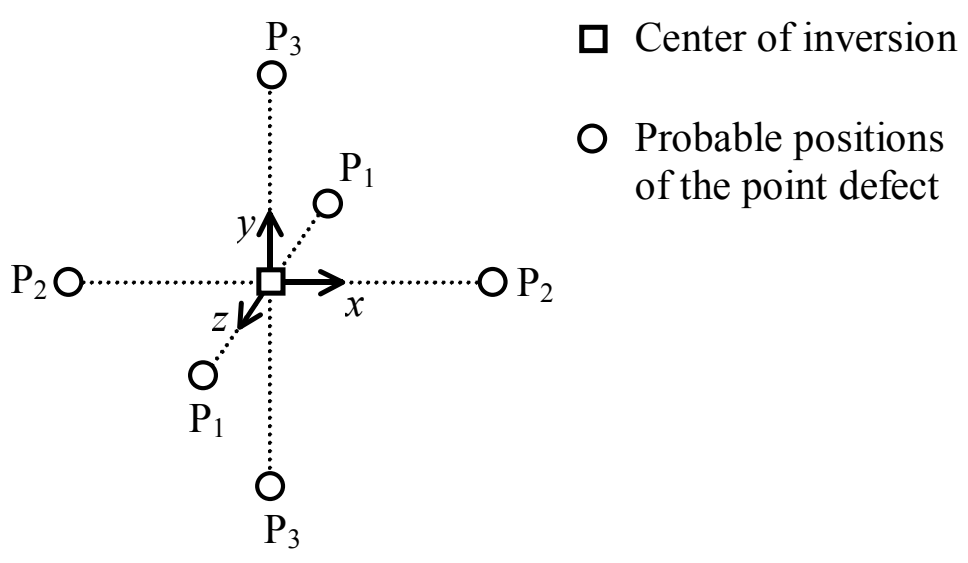

Fig. 1. An example of nonequilibrium statistical distribution of point defects in the cubic crystal.

Table 1. Transformation of probabilities and stress tensor components by generating symmetry operations of cubic group $m 3 m$.

\begin{tabular}{|c|ccc|ccc|}
\hline & $P_{1}$ & $P_{2}$ & $P_{3}$ & $\varepsilon_{x x}$ & $\varepsilon_{y y}$ & $\varepsilon_{z z}$ \\
\hline$\hat{\mathrm{I}}$ & $P_{1}$ & $P_{2}$ & $P_{3}$ & $\varepsilon_{x x}$ & $\varepsilon_{y y}$ & $\varepsilon_{z z}$ \\
$4_{y}$ & $P_{3}$ & $P_{2}$ & $P_{1}$ & $\varepsilon_{z z}$ & $\varepsilon_{y y}$ & $\varepsilon_{x x}$ \\
$4_{z}$ & $P_{2}$ & $P_{1}$ & $P_{3}$ & $\varepsilon_{y y}$ & $\varepsilon_{x x}$ & $\varepsilon_{z z}$ \\
\hline
\end{tabular}

As it is seen from the table, the transformational roles for probabilities grades into those for strain tensor components after the replacement of $P_{1}, P_{2}$ and $P_{3}$ by $\varepsilon_{x x}, \varepsilon_{y y}$, and $\varepsilon_{z z}$, respectively. As so, the basic functions of one- and two-dimensional irreducible representations of cubic grope can be composed of probabilities by the analogy with the functions $u_{1}$ and $u_{2,3}$. Therefore, the components of slow order parameter may be expressed as

$$
\eta_{1}=r_{A}\left(3 P_{0}-P_{1}-P_{2}-P_{3}\right) / 3, \eta_{2}=\sqrt{3} r_{A}\left(P_{1}-P_{2}\right), \eta_{3}=r_{A}\left(2 P_{3}-P_{2}-P_{1}\right),
$$

where $r_{A}<<1$ is the relative number of positions occupied by defects. According to cubic symmetry, $P_{1}=P_{2}=P_{3} \equiv P_{0}=1 / 3$ in the equilibrium. Therefore, the equilibrium values of slow order parameter components are equal to zero in the austenitic phase. For the every variant of martensitic phase the slow order parameter components are interrelated with MT strains by Eq. 5 .

\section{Computations}

Input Data. All computations were carried out for $\mathrm{Ni}-\mathrm{Mn}-\mathrm{Ga}$ alloy with $\mathrm{MT}$ temperatures close to $202 \mathrm{~K}$ [8]. For this alloy the temperature values $T_{1}=203.5 \mathrm{~K}$ and $T_{2}=201 \mathrm{~K}$ [9] were obtained. The coefficients $a_{4}=26 \mathrm{GPa}$ and $b_{4}=509 \mathrm{GPa}$ were evaluated in [9] from the temperature dependencies of lattice parameters and shear elastic modulus of cubic phase. The volume change $\Delta V / V \equiv\left[V_{A}-V_{M}(0)\right] / V_{A}=-u_{1} / 3$ and lattice distortion $\left[1-c\left(T_{2}\right) / a\left(T_{2}\right)\right]=u_{3} / 2$ caused by MT were estimated from the experimental data reported in $[7,8]$. The values used for computations are shown in the Table 2. The parameter $a_{2}$ is expressed through these values as $a_{2}=-2 c_{1} u_{1} / 9 u_{3}^{2}$ [7]. Due to the pronounced softening of shear modulus $C^{\prime}$ the strong inequality $C^{\prime}=\left(C_{11}-C_{12}\right) / 2<<C_{11}$ is valid, and so, $c_{1}=3\left(C_{11}+2 C_{12}\right) \approx 9 C_{11}$. 
Table 2. The values used for computations.

\begin{tabular}{|cccccc|}
\hline$u_{3}$ & $u_{1}$ & $\tau_{1,3}[\mathrm{ks}]$ & $C_{11}[\mathrm{GPa}]$ & $P^{(\mathrm{s})}(0)[\mathrm{GPa}]$ & $\sigma_{3}{ }^{(\mathrm{s})}(0)[\mathrm{MPa}]$ \\
\hline 0.052 & $-2.8 \times 10^{-3}$ & 40 & 200 & $0.64^{1)}$ & $-3^{2)}$ \\
\hline
\end{tabular}

1) This value provides for the experimental magnitude of MT temperature shift.

${ }^{2)}$ This is the tentative value; its choice is explained in the text.

Results. The theoretical time dependencies of SP and SAS are presented in Fig. 2 (a). The choice of abnormally low value $\sigma_{3}{ }^{(\mathrm{s})}(0)$ is suggested by the following facts. For the single crystalline Ni$\mathrm{Mn}-\mathrm{Ga}$ sample trained by cyclic magnetic field the yield (twinning) stress is of about $2 \mathrm{MPa}$ [10]. The aging of the sample at room temperature results in the multiplication of this value by factor 2 or 3.

The computed time dependencies of MT temperature shifts caused by martensite aging are presented in Fig. 2 (b). The dashed line illustrates the martensite stabilization effect caused by SP, solid one shows the total value of MT temperature shift $\Delta T_{M}=\Delta T_{M}^{i s o t r}+\Delta T_{M}^{\text {anis }}$, which characterizes the stabilization of martensite during its aging.

The quick and slow order parameters are interrelated linearly. Therefore, the martensite aging must be accompanied by the slow deformation of crystal lattice, as it was observed in [11]. In particular, the slow evolution of the martensite volume

$$
\Delta V(t) / V=(\Delta V / V)+9 P^{(s)}(t) / c_{1}
$$

must take place. The value Eq. 13 was computed using the theoretical time dependence of SP (Fig. 2 (a)), involved in the term, which expresses the elastic compression of martensite by SP. The result of computation is shown in Fig. 2 (c).
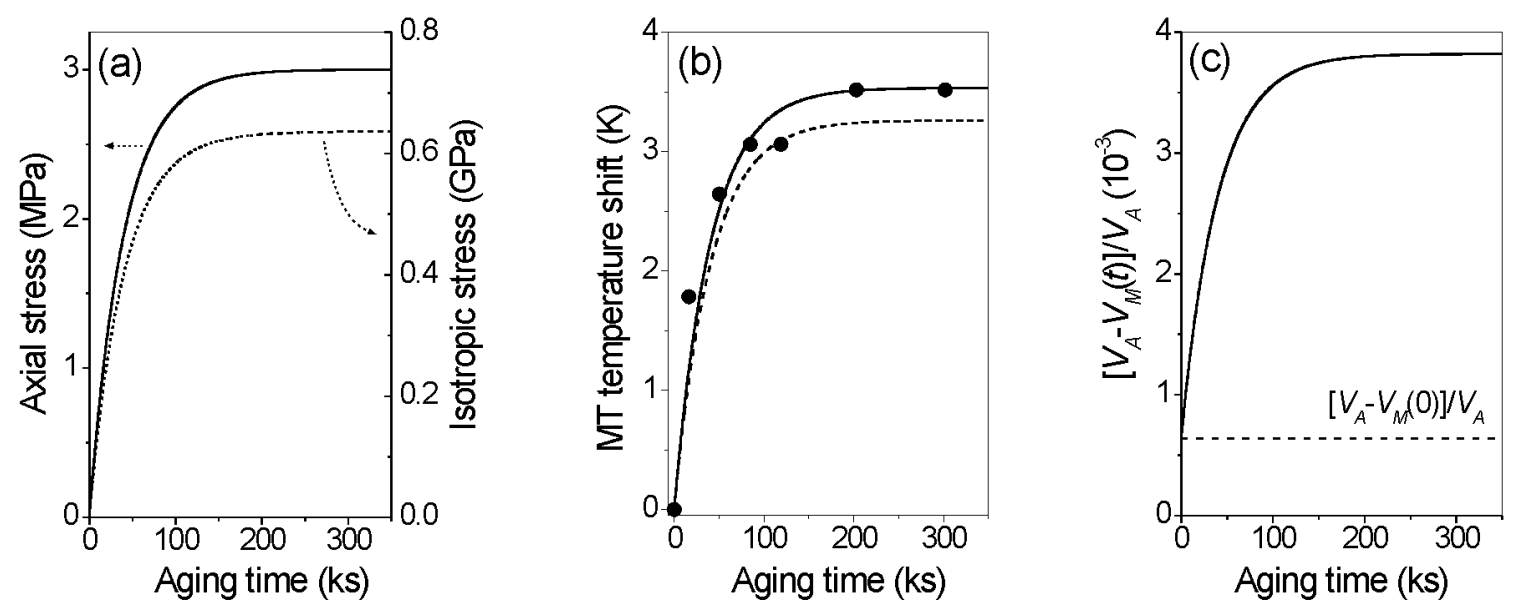

Fig. 2. Theoretical time dependencies of isotropic (dashes) and axial (solid) parts of stabilizing internal stress, (a), shift of MT temperature, (b) and volume, (c), during the martensite aging. Experimental values [12] are shown by circles.

The time dependence of volume change suggests an idea that the martensite aging can be one of the reasons of discrepancy between the $\Delta V(t) / V$ values estimated for the similar alloys by different methods [7]. The value estimated from Clausius-Clapeyron relationship is close to initial value $\Delta V(0) / V$, because the latent heat of $\mathrm{MT}$, which is involved in this relationship, is measured during MT. The volume change evaluated from the lattice parameters may exceed the thermodynamic estimation substantially if the values of these parameters are measured long after MT. 


\section{Conclusions}

According to the Principle Requirement, which was assumed as the basis of the theory developed above, the slow reconfiguration of defects in the crystal can be described by the multicomponent time-dependent order parameter. The Principle Requirement is necessary for the analytic description of the process of symmetry conforming reconfiguration of crystal defects after the MT. The Landau theory with multicomponent order parameter results in the following conclusions.

1. The martensite aging is contributed by slow reconfiguration of the every sort of defects distorting the crystal lattice and inducing the Stabilizing Internal Stress.

2. The SIS can be subdivided into the Stabilizing Axial Stress and Stabilizing Pressure. The SAS causes the increase of yield stress after the martensite aging while the observed increase of MT temperature is caused by superposition of SAS and SP. As so, the magnitude relation between these two effects may be different for different alloys: the alloy with pronounced increase of MT temperature may exhibit slight increase of yield stress and vice versa.

3. The strain tensor components are linearly related to the components of slow order parameter, and due to this the martensite aging is accompanied by the slow deformation of crystal lattice (as it was observed in [11]).

4. The introduction of SIS conception as the universal origin of martensite stabilization bridges a gap between the general approaches to the problem solution and consideration of the roles of different defects. To this end the crystal distortions and microstresses created by these defects would be described using the existing models of point defects and dislocations.

\section{Acknowledgement}

V.A.L. is grateful to Academy of Finland for the support of his stay in Helsinki University of Technology.

\section{References}

[1] X. Ren and K. Otsuka: Nature Vol. 389 (1997), p. 579.

[2] X. Ren and K. Otsuka, Phys. Rev. Lett. Vol. 85 (2000), p. 1016.

[3] T. Okuzono, Y. Yamazaki and T. Ohta: Phys. Rev. B Vol. 67 (2003), p. 054106.

[4] Y. Murakami: Journ. Phys. Soc. Japan Vol. 38 (1975), p. 404.

[5] G. R. Barsch: Phys. Rev. Lett. Vol. 53 (1984), p. 1069.

[6] E.V. Gomonaj and V.A. L'vov: Phase Transitions Vol. 47 (1994), p. 9.

[7] V.A. Chernenko and V.A. L'vov: Phil. Mag. A Vol.73 (1996), p. 999.

[8] Y. Ma, S. Awaji, K. Watanabe, M. Matsumoto and N. Kobayashi: Solid State Commun. Vol. 113 (2000), p. 671.

[9] V.A. L'vov, N. Glavatska, I. Aaltio, O. Sцderberg, I. Glavatskyy and S-P. Hannula: accepted to Acta Materialia (2009).

[10] A.A. Likhachev and K. Ullakko: Physics Letters A Vol. 275 (2000), p. 142.

[11]H. Ishibashi, M. Kogachi, T. Ohba, X. Ren, and K. Otsuka: Mat. Sci. Engng. A Vol. 329-331 (2002), p. 568.

[12] V. Khovailo, R. Kainuma, T. Abe, K. Oikawa and T. Takagi: Scripta Mat. Vol. 51 (2004), p.13. 